\title{
Evaluation of TeamSTEPPS integration across a curriculum regarding team attitudes: A longitudinal study
}

\author{
Mary Beth R. Maguire ${ }^{* 1}$, Marie N. Bremner ${ }^{1}$, David N. Bennett ${ }^{1}$, Lewis VanBrackle ${ }^{2}$ \\ ${ }^{1}$ WellStar School of Nursing, Kennesaw State University, Kennesaw, Georgia, United States \\ ${ }^{2}$ Department of Statistics and Analytical Sciences, Kennesaw State University, Kennesaw, Georgia, United States
}

Received: March 17, 2015

DOI: $10.5430 /$ jnep.v5n7p131

\author{
Accepted: April 7, 2015 \\ Online Published: May 11, 2015 \\ URL: http://dx.doi.org/10.5430/jnep.v5n7p131
}

\begin{abstract}
Introduction: The ability to function as an effective member of the healthcare team is essential for graduate nurses upon entry into practice. Team training is therefore, an important element of nursing education. A convenient and cost-effective approach to providing team training to nursing students is through the TeamSTEPPS ${ }^{\circledR}$ curriculum. The purpose of the study was to determine if an intentional integration of TeamSTEPPS ${ }^{\circledR}$ principles into simulation-based team training modules would improve attitudes toward teamwork in a cohort of undergraduate nursing students.

Methods: A quasi-experimental time series nonequivalent control group design was used. A convenience sample of 115 first semester students (108 completed) who received the TeamSTEPPS ${ }^{\circledR}$ training and 77 final semester undergraduate students who did not receive the intervention participated. Repeated measures of the TeamSTEPPS-Teamwork Attitude Questionnaire (T-TAQ) were obtained initially and three times throughout the curriculum. Final semester students served as the comparison group and completed the T-TAQ without formal team training.

Results: After participation in ten hours of simulation-based instructional activities, T-TAQ scores significantly increased from baseline and maintained over time. No statistical difference was identified between first semester students without formal team training and graduating students without formal team training.

Conclusions: The findings suggest an intentional integration of TeamSTEPPS ${ }^{\circledR}$ principles throughout an undergraduate-nursing curriculum improve and maintain student teamwork attitudes over time. It is recommended that TeamSTEPPS ${ }^{\circledR}$ principles be intentionally integrated throughout undergraduate nursing curricula.
\end{abstract}

Key Words: TeamSTEPPS ${ }^{\circledR}$, Curriculum integration, Team attitudes, Nursing education

\section{INTRODUCTION}

Teams of healthcare providers, the very people entrusted by patients and families to protect and heal, are often responsible for harming and sometimes killing patients. James ${ }^{[1]}$ estimates 213,000 deaths per year are attributed to preventable adverse events (PAE's) occurring in hospitals. Deaths due to PAE's are the third leading cause of death in the United
States, behind heart disease (597,689 deaths) and cancer (574,743 deaths). ${ }^{[2]}$ The causes of PAE's can be separated into five distinct categories: errors of commission, errors of omission, errors of communication, errors of context, and diagnostic errors. ${ }^{[1]}$ Strategies that have the potential to diminish the incidence of deaths and injuries from PAE's include improving the functioning of healthcare teams and

\footnotetext{
* Correspondence: Mary Beth R. Maguire; Email: mmaguir5@kennesaw.edu; Address: WellStar School of Nursing, Kennesaw State University, Kennesaw, Georgia, United States.
} 
instituting a culture of safety into our healthcare system.

\subsection{TeamSTEPPS ${ }^{\circledR}$}

One way to improve the functioning of the healthcare team and to reduce PAE's is educating healthcare providers using the TeamSTEPPS ${ }^{\circledR}$ curriculum. TeamSTEPPS ${ }^{\circledR}$ stands for Team Strategies and Tools to Enhance Performance and Patient Safety. TeamSTEPPS ${ }^{\circledR}$ is a comprehensive set of materials and training curriculum that seeks to improve patient safety through the use of team-based principles. ${ }^{[3]}$ The TeamSTEPPS ${ }^{\circledR}$ program was created by the Agency for Healthcare Research and Quality (AHRQ) and the Department of Defense (DOD) in response to the rising tide of healthcare errors. The curriculum is an evidence-based program based on 25 years of research related to teamwork, team training, and culture change. ${ }^{[3]}$ TeamSTEPPS ${ }^{\circledR}$ was adopted as the national standard for healthcare team training in November 2006. ${ }^{[14]}$ In 2014 the program was updated and renamed TeamSTEPPS ${ }^{\circledR} 2.0$ to include: a greater emphasis on communicating early and often to improve teamwork, a course management guide, and a measurement module. ${ }^{[5]}$ This study was conducted using the original TeamSTEPPS ${ }^{\circledR}$ curriculum.

The TeamSTEPPS ${ }^{\circledR}$ program is comprised of four primary teamwork skills: leadership, communication, situation monitoring, and mutual support. The TeamSTEPPS ${ }^{\circledR}$ curriculum reinforces the use of behaviors such as Situation-BackgroundAssessment-Recommendation (SBAR), check-back, and huddle which seek to improve team performance. ${ }^{[3]}$ The program prepares team members to question decisions in patient care situations and support one another without assigning blame. The implementation of TeamSTEPPS ${ }^{\circledR}$ principles has proven to reduce negative patient outcomes. ${ }^{[6]}$ One hospital reported a $30 \%$ reduction in medical errors and an $88 \%$ decrease in the number of patient falls after implementing TeamSTEPPS ${ }^{\circledR}$ training. ${ }^{[7]}$

The simulated clinical experience provides an ideal opportunity for learners to practice and refine clinical skills, teamwork, and communication in a supervised, controlled environment using a set of pre-determined objectives. ${ }^{[8]}$ Combining simulation and the TeamSTEPPS ${ }^{\mathrm{R}}$ curriculum has the potential to be an effective teaching strategy to allow learners the opportunity to engage in experiences addressing knowledge, skill, and interpersonal interactions while practicing team strategies in a safe, reproducible environment.

\subsection{Literature review}

While most professional accrediting bodies encourage student educational experiences in interdisciplinary teams, the concepts of how teams work together effectively to achieve 132 safety and successful patient outcomes have not consistently been a clear focus in nursing curricula. The value of interprofessional education (IPE) is well documented in the literature $^{[9]}$ and commonalities and differences among the attitudes of health science students have been identified. ${ }^{[10]}$ However, there is a need to assess the quality of teamwork content across the nursing curriculum and strategies to improve teamwork outcomes. An additional concern is that not all Schools of Nursing have the availability and opportunity to connect with other professions to conduct IPE.

Although a value of IPE is well documented ${ }^{[11]}$ studies of attitude are mostly descriptive in nature, with a focus on differentiating attitudes of students of medicine, nursing, pharmacy and social work ${ }^{[9,10]}$ and faculty versus student attitudes. ${ }^{[12]}$ These cross sectional, descriptive studies do not measure the duration of change in attitudes or if the initial change results in future behavioral change.

The use of a standardized curriculum such as TeamSTEPPS ${ }^{\circledR}$ and measurements using the TeamSTEPPSTeamwork Attitude Questionnaire (T-TAQ) is a means of standardizing the measurement of attitude change. Several studies measuring teamwork were found but none of these incorporated the use of a standardized curriculum. The only longitudinal study that included health science students to include: medicine, nursing, pharmacy, and social work described attitudes toward teamwork and interprofessional education was a study that covered a three year period from 2005 - 2007, however this research did not specifically use TeamSTEPPS ${ }^{\mathrm{R}}$. [10]

Limited research exists on the longitudinal effect of the introduction of TeamSTEPPS ${ }^{\circledR}$ concepts in undergraduate students' attitude toward teamwork. A limited number of studies were identified that did assess changes after TeamSTEPPS ${ }^{\circledR}$ implementation however these described cross sectional results rather than longitudinal changes. Vertino ${ }^{[13]}$ evaluated nursing staff to include Registered Nurses, Licensed Practical Nurses, and Nursing Assistants attitudes toward teamwork using the T-TAQ. Caylor, Aebersond, Lapham \& Carlson ${ }^{[14]}$ modified a TeamSTEPPS ${ }^{\circledR}$ training with use of a virtual simulation for 21 nursing, medicine and pharmacy students. The T-TAQ was assessed one week before the simulation and immediately following intervention. Overall, these studies support the need for a longitudinal assessment of a formal TeamSTEPPS ${ }^{\circledR}$ integration among undergraduate nursing students.

\subsection{Research questions}

The purpose of the study was to determine if an intentional integration of TeamSTEPPS ${ }^{\circledR}$ principles into simulation- 
based team training modules would improve attitudes toward teamwork in a cohort of undergraduate nursing students. The study proposed the following questions:

(1) Does TeamSTEPPS ${ }^{\circledR}$ training with undergraduate nursing students across the curriculum improve students' attitudes toward teamwork?

(2) Are there differences in attitudes toward teamwork of first semester students prior to formal TeamSTEPPS ${ }^{\circledR}$ training and final semester students without formal TeamSTEPPS ${ }^{\circledR}$ training?

(3) Are there differences in attitudes toward teamwork of first semester students with formal TeamSTEPPS ${ }^{\circledR}$ training to final semester students without formal TeamSTESSP ${ }^{\mathrm{R}}$ training?

\section{MeTHODS}

A quasi-experimental time series nonequivalent control group design was used. A convenience sample of 115 first semester students (108 completed) who received the TeamSTEPPS ${ }^{\circledR}$ training and 77 final semester undergraduate students who did not receive the intervention were invited to participate in the study. First semester students participated in 10 hours of training at designated points throughout the nursing curriculum. Repeated measures of the T-TAQ were obtained initially and three times throughout the curriculum. Final semester students served as the comparison group and completed the T-TAQ without formal team training. The Institutional Review Board approved the project. Written informed consent, including assurance of privacy and confidentiality of responses, was obtained from each participant.

\subsection{Project implementation/TeamSTEPPS ${ }^{\circledR}$ curricu- lum mapping}

A demographic questionnaire and baseline T-TAQ was completed by undergraduate nursing students enrolled in a first semester clinical course prior to receiving 6 hours of TeamSTEPPS modules within a simulation center. The TeamSTEPPS ${ }^{\circledR}$ Essentials curriculum was divided over 2 days of scheduled simulations. Day 1 was held the week prior to the first in-hospital clinical experience with a 2-hour didactic session and instructor facilitated discussions that emphasized the development of Situational Monitoring and Mutual Support skills. Day 2 was held the week after first semester clinical experiences were completed. This fourhour session reviewed the team skills of Situational Monitoring and Mutual Support and introduced new concepts of team leadership and team communication. Groups of 3-4 students completed a low fidelity scenario with a focus on reducing the risk of health care associated infections. Students were debriefed after the simulation with instructor guidance. At the end of this course, students completed the first of three post assessments using the T-TAQ.

In the second semester, participating students engaged in a one hour, low fidelity scenario appropriate for an adult health nursing clinical course. Following a brief review of TeamSTEPPS ${ }^{\circledR}$ principles and framework, two specific tools were emphasized in the Mutual Support category. The Two-Challenge rule calls for the team member being challenged to acknowledge the concern. If the assertion is ignored after two attempts, the concerned team member is instructed to take a stronger course of action or utilize the chain of command to intervene. CUS stands for I'm Concerned, I'm Uncomfortable, and this is a Safety issue. The CUS strategy is one way of employing the Two-Challenge rule. The team member being challenged is responsible to address the concern. Other specific tools were emphasized in the Communication category to include: SBAR, Call-outs and Check-backs. SBAR is a technique to communicate official information that requires immediate attention and action concerning a patient's condition. S stands for situation, or what is going on with the patient. B stands for background, or what is the relevant clinical history. A stands for assessment, or what the problem is thought to be. $\mathrm{R}$ stands for recommendation, or what can be done to correct the situation. Call-outs are a strategy used to communicate important or critical information by informing team members simultaneously and help team members anticipate the next steps. Check-backs serve as a process of closed-loop communication to ensure information conveyed by the sender is understood by the intended receiver. The sender double-checks to ensure that the message was received.

Students viewed the influential video of Susan Sheridan ${ }^{[15]}$ whose family was tragically affected by the result of poor medical team performance. Students were then asked to demonstrate Mutual Support within a similar Perioperative Simulated Experience. After viewing vignettes provided in the TeamSTEPPS ${ }^{\circledR}$ curriculum regarding caring for a female patient in a hypothyroid crisis, students were asked to role-play the introduced communication techniques within a simulated medical unit. At the conclusion of both scenarios, students participated in a debriefing session with faculty.

In the third semester, a high fidelity scenario was incorporated within the parent and child health clinical nursing course. The two-hour module began with a discussion of students' experiences and their perceptions after observing and/or participating the first two semesters in TeamSTEPPS ${ }^{\circledR}$. The purpose of this discussion was not only to review TeamSTEPPS ${ }^{\circledR}$ but also to give an opportu- 
nity for students to share their unique experiences. Specific tools and strategies highlighted during this session were Brief (similar to a flight checklist), Huddle (ad hoc planning sessions), Debrief (once an event has taken place to review what went well and what changes can occur to improve performance), and Two-Challenge Rule.

Following this discussion, students had the opportunity to apply what they learned in the classroom and clinical setting regarding the care of a patient with Postpartum Hemorrhage. Laerdal SimMom and the Postpartum Hemorrhage scenario were used for this high fidelity scenario. Students in two groups of five were given a detailed report and expected to conduct a comprehensive, hands on assessment, recognize an emergency, implement priority interventions, assemble a team, and notify a health provider.

The classmates of the students involved in the simulation viewed the simulation scenario via real-time streaming video into the classroom. During and immediately after the simulation, peers utilized an automatic response device (i>clicker) to document specific TeamSTEPPS ${ }^{\circledR}$ strategies observed in the simulation. The "clicker" technology was included in the simulation-based team training to improve engagement among all participants. Following the 20-minute live simulation, all students convened in the classroom for a 40 minute debrief session.

In the final semester of the curriculum students participated in the last hour of the 10 hour TeamSTEPPS ${ }^{\circledR}$ curriculum. Following a brief recap of previous teamwork sessions, students applied the concepts of TeamSTEPPS ${ }^{\circledR}$ to team error disclosure. A variety of low fidelity scenarios were developed relating to the distinct categories of PAE's that James ${ }^{[1]}$ identified. Students in teams were required to plan a team disclosure for errors of commission, omission, communication, context and diagnostic categories. Following the debrief session, students completed the final observation T-TAQ to measure their attitude toward teamwork. Upon completion of the tool, each student received a certificate of completion for the TeamSTEPPS Essentials Curriculum.

\subsection{Framework}

A commonly employed theoretical framework used in the study of healthcare team training is Kirkpatrick's four-level model of evaluation. ${ }^{[16]}$ The Kirkpatrick model is often used because it is perhaps the best-known evaluation methodology for assessing learning processes. While most people refer to the four criteria for evaluation of learning processes as "levels" Kirkpatrick calls them "steps" ${ }^{[17]}$ The first step is reaction. The second step is learning. The third step is behavior. The fourth and final step of Kirkpatrick's model is results. The use of the Kirkpatrick model was an appropriate first step in exploring the effectiveness of TeamSTEPPS ${ }^{\circledR}$ implementation across a curriculum.

\subsection{Tool}

The 30-item T-TAQ (available from: http://teamstep ps/ahrq.gov/taq_index.htm) was utilized to measure participants' attitudes toward the core components of teamwork in healthcare. The T-TAQ provides data to evaluate 5 core team competencies: Team Structure, Leadership, Mutual Support, Situation Monitoring, and Communication using a 5-point Likert scale $(1=$ strongly disagree, $5=$ strongly agree). Cronbach's alpha coefficient of internal consistency reliability for the teamwork constructs are reported as $.70, .81, .83, .70$, and .74 , respectively (http://teamstepps.ahrq.gov/taq_index.htm). The instrument can be administered as a stand-alone assessment or to evaluate changes in team attitudes over time.

\subsection{Data analysis}

Data were analyzed using JMP Statistical Software, Version 11. A control group of 77 graduating seniors were queried at the end of the program without formal TeamSTEPPS training. One cohort of 115 students consented to participate in the study with 108 students completing the study. Study participant demographics can be found in Table 1 .

Table 1. Sample demographics

\begin{tabular}{lll}
\hline & Control $(\mathbf{N}=\mathbf{7 7})$ & Participant $(\mathbf{N}=\mathbf{1 0 8})$ \\
\hline \multirow{2}{*}{ Age } & 21-58 range years & 18-53 range years \\
& 21.58 mean & 27.5 mean \\
& 11 males & 15 males \\
& 66 females & 93 females \\
& & 74 White/Caucasian \\
& 56 White/Caucasian & 12 Black/African \\
Ethnicity & 9 Black/African American & American \\
& 7 Asian or Pacific Islander & 11 Asian or Pacific \\
& 1 Hispanic/Latino & Islander \\
& 6 Other & 9 Hispanic/Latino \\
Program & 61 Traditional & 2 Other \\
Enrolled & 16 Accelerated & 75 Traditional \\
\hline
\end{tabular}

\section{RESULTS}

\subsection{As related to Q1: Does TeamSTEPPS ${ }^{\circledR}$ training with undergraduate nursing students across the cur- riculum improve students' attitudes toward team- work?}

To assess the effect of TeamSTEPPS ${ }^{\circledR}$ training with undergraduate nursing students across the curriculum a repeated measures Analysis of Variance was used to test for differences of population means scores for all five subscales across 
the three time periods. There were statistically significant differences across the three time periods for the Team Structure subscale $[\mathrm{F}(2,168)=3.90, p=.022)]$ and Situation Monitoring subscale $[\mathrm{F}(2,168)=3.94), p=.021)]$.

For both the Team Structure and Situation Monitoring subscale, Tukey's HSD test found there to be significant differences between the end of semester and beginning of semester means. For the Team Structure subscale, the mean score for the end of the semester was significantly larger than the mean score at the end of semester. For the Situation Monitoring subscale, the effect was reversed with the mean score for the end of the semester significantly smaller than the mean score at the end of semester. The instrument is inversely scored for this variable.

Table 2 shows the sample means of the 115 treatment participants for the five subscales across the three time periods.

Table 2. Sample means of subscales for treatment group

\begin{tabular}{llllll}
\hline Time & Team Structure & Leadership & Situation Monitoring & Mutual Support & Communication \\
\hline Baseline & 4.48 & 4.70 & 4.49 & 4.47 & 4.52 \\
Midpoint & 4.65 & 4.74 & 4.70 & 4.37 & 4.55 \\
End of Program & 4.72 & 4.84 & 4.75 & 4.28 & 4.54 \\
\hline
\end{tabular}

3.2 As related to Q2: Are there differences in attitudes toward teamwork of first semester students prior to formal TeamSTEPPS ${ }^{\circledR}$ training and final semester students without formal TeamSTEPPS ${ }^{\circledR}$ training?

To assess differences in attitudes toward teamwork between first semester students prior to formal TeamSTEPPS ${ }^{\circledR}$ training and the control group of final semester students without formal TeamSTEPPS ${ }^{\circledR}$ training, two-sample $t$-tests were conducted for each subscale. Comparing first semester students prior to formal TeamSTEPPS ${ }^{\circledR}$ training and final semester students without formal TeamSTEPPS ${ }^{\mathbb{R}}$ training. As shown in Table 3, there were no statistically significant differences of mean subscale scores between first semester students prior to formal TeamSTEPPS ${ }^{\circledR}$ training and final semester students without formal TeamSTEPPS ${ }^{\circledR}$ training.
Table 3. First semester students prior to formal TeamSTEPPS ${ }^{\circledR}$ training versus final semester students without formal TeamSTEPPS ${ }^{\circledR}$ training

\begin{tabular}{llll}
\hline Subscale & $\boldsymbol{t}$ statistic & df & $\boldsymbol{p}$-value \\
\hline Team Structure & -1.34 & 183 & 0.183 \\
Leadership & 0.68 & 183 & 0.498 \\
Situation Monitoring & 0.83 & 183 & 0.407 \\
Mutual Support & 0.87 & 183 & 0.385 \\
Communication & -0.39 & 183 & 0.700 \\
\hline
\end{tabular}

The sample means and standard deviations for the subscales for first semester students prior to formal TeamSTEPPS ${ }^{\circledR}$ training and final semester students without formal TeamSTEPPS ${ }^{\circledR}$ training are shown in Table 4.

Table 4. First semester students prior to formal TeamSTEPPS ${ }^{\circledR}$ training and final semester students without formal TeamSTEPPS ${ }^{\circledR}$ training

\begin{tabular}{lllll}
\hline Course & Subscale & Sample Size & Mean & Standard Deviation \\
\hline \multirow{3}{*}{ First Semester } & Team Structure & 115 & 4.48 & 0.47 \\
& Leadership & 115 & 4.72 & 0.41 \\
& Situation Monitoring & 115 & 4.54 & 0.53 \\
& Mutual Support & 115 & 4.48 & 0.50 \\
& Communication & 115 & 4.54 & 0.51 \\
End of Program & Team Structure & 77 & 4.40 & 0.40 \\
& Leadership & 77 & 4.76 & 0.31 \\
& Situation Monitoring & 77 & 4.60 & 0.44 \\
& Mutual Support & 77 & 4.54 & 0.45 \\
\hline
\end{tabular}


3.3 As related to Q3: Are there differences in attitudes toward teamwork of first semester students with formal TeamSTEPPS ${ }^{\circledR}$ training to final semester students without formal TeamSTESSP ${ }^{\circledR}$ training?

To assess differences in attitudes toward teamwork of final semester students without formal TeamSTEPPS ${ }^{\circledR}$ training in comparison to first semester students with formal TeamSTEPPS ${ }^{\circledR}$ training, two-sample t-tests were conducted for each subscale comparing final semester students without formal TeamSTEPPS ${ }^{\circledR}$ training to first semester students with formal TeamSTEPPS ${ }^{\circledR}$ training.

As shown in Table 5, there are several statistically significant differences in mean subscale scores between final semester students without formal TeamSTEPPS ${ }^{\circledR}$ training and first semester students with formal TeamSTEPPS ${ }^{\circledR}$ training.

The Team Structure and Situation Monitoring subscale means are significantly larger for the first semester students with formal TeamSTEPPS ${ }^{\circledR}$ training. The Mutual Support subscale mean is significantly larger for final semester students without formal TeamSTEPPS ${ }^{\circledR}$ training. The means for the Leadership and Communication subscales are not significantly different for final semester students without formal TeamSTEPPS ${ }^{\circledR}$ training in comparison to first semester students with formal TeamSTEPPS ${ }^{\circledR}$ training.

Table 5. Firs semester students with formal TeamSTEPPS ${ }^{\circledR}$ training and final semester students without formal TeamSTESSP ${ }^{\circledR}$ training

\begin{tabular}{llll}
\hline Subscale & $\boldsymbol{t}$ statistic & df & $\boldsymbol{p}$-value \\
\hline Team Structure & 4.67 & 191 & $<.001$ \\
Leadership & 1.22 & 191 & .225 \\
Situation Monitoring & 2.45 & 191 & .015 \\
Mutual Support & -3.20 & 191 & .002 \\
Communication & -0.10 & 191 & .924 \\
\hline
\end{tabular}

The sample means and standard deviations of the subscales for final semester students without formal TeamSTEPPS ${ }^{\circledR}$ training and first semester students with formal TeamSTEPPS ${ }^{\circledR}$ training are shown in Table 6 .

Table 6. Final semester students without formal TeamSTEPPS training versus final semester with formal TeamSTEPPS training

\begin{tabular}{|c|c|c|c|c|}
\hline Course & Subscale & Sample Size & Mean & Standard Deviation \\
\hline \multirow{5}{*}{$\begin{array}{l}\text { Graduating Seniors Without Formal } \\
\text { TeamSTEPPS Training }\end{array}$} & Team Structure & 77 & 4.40 & 0.40 \\
\hline & Leadership & 77 & 4.76 & 0.31 \\
\hline & Situation Monitoring & 77 & 4.60 & 0.44 \\
\hline & Mutual Support & 77 & 4.54 & 0.45 \\
\hline & Communication & 77 & 4.51 & 0.40 \\
\hline \multirow{5}{*}{$\begin{array}{l}\text { Graduating Seniors With Formal } \\
\text { TeamSTEPPS Training }\end{array}$} & Team Structure & 108 & 4.65 & 0.35 \\
\hline & Leadership & 108 & 4.81 & 0.30 \\
\hline & Situation Monitoring & 108 & 4.74 & 0.34 \\
\hline & Mutual Support & 108 & 4.24 & 0.74 \\
\hline & Communication & 108 & 4.50 & 0.45 \\
\hline
\end{tabular}

\section{DISCUSSION}

The study findings indicate that TeamSTEPPS training resulted in improved attitudes toward teamwork. This finding supports Kirkpatrick's ${ }^{[16]}$ first step of the four level model of evaluation in that there was evidence of a positive reaction to team attitudes. Data analysis suggests that the greatest gain in student attitudes was achieved in the first semester after introduction of the Essentials curriculum with augmented simulation experiences. The results indicate that the TeamSTEPPS ${ }^{\circledR}$ training did improve attitudes toward teamwork but that the largest gain was found in the initial six hours of content. The additional four hours of content helped to maintain the initial improvement in attitudes among these students. An unexpected finding was the similarity between teamwork attitudes between first semester student's pre intervention with no formal TeamSTEPPS ${ }^{\circledR}$ training and final semester students with no formal TeamSTEPPS ${ }^{\circledR}$ training. This finding could indicate a need for curriculum review for the introduction and implementation of team concepts. A possibility is that the terminology of TeamSTEPPS ${ }^{\circledR}$ may not be the same as the concepts introduced in the curriculum.

TeamSTEPPS ${ }^{\circledR}$ concepts were easily integrated within current course and clinical objectives in both classroom and practice laboratory setting. The TeamSTEPPS ${ }^{\circledR}$ concepts align with accreditation mandates thus the current curricu- 
lum was already addressing these concepts from a broad perspective. However, it was through active engagement in the classroom, laboratory and simulation environment that greater attention was called to the TeamSTEPPS ${ }^{\circledR}$ concepts. The student engagement allowed for rehearsing behaviors and presentation of a standardization of language to better equip students to transition to the practice environment. The free TeamSTEPPS ${ }^{R}$ curriculum is robust and easily accessible to include video vignettes, PowerPoint slides, discussion points, and evaluation materials (found at http: //teamstepps.ahrq.gov/).

\section{Limitations}

A limitation of the study is that student involvement was voluntary and not made a part of course requirements. The idea for a pilot study was initially introduced after semester schedules had already been developed. The training and the study were conducted during time periods after examinations or after classes were complete for the day. Student participation could have been increased, and perhaps improved upon, if the researchers had solicited faculty buy in and spent more time explaining the key concepts of TeamSTEPPS ${ }^{\circledR}$. As it was, faculty members did not feel the need for change as strongly as the researchers and were less likely to accommodate the training and attitude testing during regular didactic or clinical settings. Presentation of concepts in time periods not related to a specific class could have resulted in students not valuing these concepts as much as specific classroom topics.

The faculty at this university is large, experienced, and possess a diverse range of interest. With this diversity of interest comes the challenge of finding a shared vision as well as creating a sense of urgency for change to a team based approach to patient safety. This limitation is consistent with Kotter's ${ }^{[18]}$ eight-step change model that recommends the investment of time in addressing buy in by the faculty and a shared vision for change. If others are considering implementing TeamSTEPPS ${ }^{\circledR}$, time invested in onboarding key faculty and administrators could increase student buy in as well.
Another study limitation is that students often work in local health systems as interns on a part-time basis after completion of their second semester of nursing school. There was no way to control the type of formal or informal training in TeamSTEPPS ${ }^{\circledR}$ or other patient safety initiatives. To some extent, the attitude changes measured could be influenced by exposure to these concepts in the work setting.

\section{Conclusion}

Nurse educators have a responsibility to prepare new graduates to function as competent and effective team members. Developing team-based simulations for nursing students offer not only a risk-free environment to practice team skills but the opportunity to participate in creating a culture shift to teamwork. In accord with the 2006 National Implementation plan, it is the responsibility of healthcare educators to address issues related to contemporary practice and prepare graduates with TeamSTEPPS ${ }^{\circledR}$ skills.

A culture of safety is an important standard in every healthcare setting. Attitude change among providers is an essential first step. The findings support that attitude changes were realized upon conclusion of the program. Evidence suggests repeated exposure to TeamSTEPPS ${ }^{\circledR}$ strategies can change and sustain positive attitudes toward teamwork and improve the culture of safety.

While beyond the scope of this study, future TeamSTEPPS ${ }^{\circledR}$ initiatives are needed in both education and practice to document the impact teamwork attitudes have on team performance, patient safety, employee satisfaction/retention, and patient satisfaction. Future projects are particularly needed to document reliable and valid tools to measure team performance and long-term implications of related outcomes. Such work holds the promise to decrease PAE's and increase the quality of care provided leading to a safer healthcare environment for all.

\section{CONFlicts OF INTEREST Disclosure}

The authors declare that there is no conflict of interest statement.

\section{REFERENCES}

[1] James JT. A new, evidence-based estimate of patient harms associated with hospital care. Journal of Patient Safety. 2013; 9(3): 122128. PMid:23860193 http://dx.doi .org/10.1097/PTS.0b013 e3182948a69

[2] Centers for Disease Control and Prevention. Leading causes of death. 2013. Available from: http://www.cdc.gov/nchs/fastats/l

Published by Sciedu Press cod.htm

[3] Agency for Healthcare Research and Quality. TeamSTEPPS: National Implementation. 2013. Available from: http://teamstepps . ahrq.gov

[4] American Institutes for Research. TeamSTEPPS teamwork perceptions questionnaire manual. 2010. Available from: http://teamstepps.ahrq.gov/teamowrk_perception_qu 
estionnaire.pdf

[5] PR Newswire. TeamSTEPPS 2.0 updates, streamlines well-known patient safety training curriculum. 2014.

[6] Courtright SH, Stewart GL, Ward MM. Applying research to save lives. Organizational Dynamics. 2012; 41(4): 291-302.

[7] Nailberk D. Hospital cuts med errors $30 \%$, falls $88 \%$ with TeamSTEPPS. Healthcare Risk Management. 2012; 90-91. http://dx.d oi .org/10.1016/j.orgdyn.2012.08.004

[8] Clapper TC, Kong M. TeamSTEPPS: The patient safety tool that needs to be implemented. Clinical Simulation in Nursing. 2012; 8 : 367-373. http://dx.doi.org/10.1016/j.ecns.2011.03.002

[9] Delunas LR, Rouse S. Nursing and medical student attitudes about communication and collaboration before and after an interprofessional education experience. Nursing Education Perspectives. 2014 35(2): 100-105. PMid:24783725 http://dx.doi.org/10.5480 /11-716.1

[10] Curran VR, Sharpe D, Forristall J, et al. Attitudes of health sciences students towards interprofessional teamwork and education. Learning in Health and Social Care. 2012; 7(3): 146-156. http: //dx.doi.org/10.1111/j.1473-6861.2008.00184.x

[11] Aase I, Aase K, Dieckmann P. Teaching interprofessional teamwork in medical and nursing education in Norway: A content analysis. Journal of Interprofessional Care. 2013; 27: 238-245. PMid:23205762 http://dx.doi.org/10.3109/13561820.2012.745489
[12] Hoffman J, Redman-Bentley D. Comparison of faculty and student attitudes toward teamwork and collaboration in interprofessional education. Journal of Interprofessional Care. 2012; 26: 6668. PMid:22233371 http://dx.doi.org/10.3109/13561820. 2011.602441

[13] Vertino KA. Evaluation of a TeamSTEPPS initiative on staff attitudes toward teamwork. The Journal of Nursing Administration. 2014; 44(2): 97-102. PMid:24451447 http://dx.doi.org/10.1097/N NA. 0000000000000032

[14] Cayler S, Aeberson M, Lapham J, et al. The use of virtual simulation and a modified TeamSTEPPS training for multiprofessional education. Clinical Simulation in Nursing. 2015; 11: 163-171. http://dx.doi.org/10.1016/j.ecns.2014.12.003

[15] World Health Organization. Patient safety interview: Susan Sheridan. 2014. Available from: http://www.who.int/patientsafety/ information_centre/interviews/Sheridan/en

[16] Kirkpatrick. Techniques for evaluating training programs. Journal of ASTD. 1959; 13: 3-9.

[17] Craig RL. The ASTD training handbook. New York: McGraw-Hill; 1996.

[18] Kotter JP. Leading change. Boston: Harvard Business School Press; 1996. 